Methods 40 male $\mathrm{BABL} / \mathrm{c}$ mice were divided into two groups, the $\mathrm{DCM}$ group and the control. The DCM group mice were peritoneal injected Coxsackievirus B3 (CVB3) monthly. After 180 days, all mice were sacrificed and IL-17 mRNA of splenocytes were measured by RT-PCR.

Results In the DCM mice, the heart weight was higher, and the ventricular wall was thinner than the control, and fibrosis in hearts were observed. IL-17 mRNA of splenocytes in DCM mice could be detected and the controls' were zero $(0.15 \pm 0.04$ vs $0.00 \pm 0.00$, $\mathrm{p}<0.01)$.

Conclusion We successfully built murine DCM model by monthly peritoneal injection of CVB3 for 180 days in the DCM group. In the DCM mice, the heart weight was higher, and the ventricular wall was thinner than the control, and fibrosis in heart was observed. The mRNA levels of IL-17 were promoted in Coxsackievirus induced DCM mice. This result suggested that IL 17 which secreted by Th17 subset could be detected in DCM mice, it seems that the Th17 cells might differentiated in DCM mice.

\section{e0152 ENDOGENOUS $\kappa$ OPIOID PEPTIDE MEDIATES THE CARDIOPROTECTION INDUCED BY ISCHAEMIC POSTCONDITIONING}

doi:10.1136/hrt.2010.208967.152

Guo Haitao, Zhang Ronghuai, Wang Yuemin, Bi Hui, Li Juan, Pei Jianming. Fourth Military Medical University

Aim Postconditioning is brief cycles of reperfusion and ischaemia during the early phase of reperfusion following a prolonged ischaemic insult. Opioids are well-known endogenous triggers of preconditioning. Because postconditioning shares the protective pathways with preconditioning, G protein-coupled receptor activation may serve as an essential mechanism that triggers protection of postconditioning. Receptor binding studies showed that $\kappa$ opioid receptor $(\kappa-O R)$ is a predominant opioid receptor in heart. Therefore, we determined whether endogenous agonist of $\kappa-\mathrm{OR}$, dynorphin, triggers postconditioning, especially reduces apoptosis of $\mathrm{I} / \mathrm{R}$ myocardium and to identify its underlying mechanism.

Methods Besides the vehicle, the other SD rats underwent a $30 \mathrm{~min}$ left anterior descending occlusion followed by $120 \mathrm{~min}$ of reperfusion with or without a postconditioning stimulus (three cycles of $10 \mathrm{~s}$ reperfusion and $10 \mathrm{~s}$ reocclusion) initiated at the onset of reperfusion. The selective $\kappa$ opioid receptor antagonist nor-binaltorphimine (Nor-BNI, $2 \mathrm{mg} / \mathrm{kg}$, intravenously), administered $5 \mathrm{~min}$ before the reperfusion. The blood plasma was analysed spectrophotometrically for determination of $\mathrm{CK}$ and $\mathrm{LDH}$ levels. Myocardial apoptosis was quantitatively analysed by detection of TUNEL with an apoptosis detection kit. Six fields from the periinfarct zone were analysed and the number of TUNEL positive cardiomyocytes was counted on 400 high power fields. Immunoreactive Dynorphin were measured by an antigen competitive ELISA. Results CK (U/L) and LDH (U/L) were significantly higher in I/R group than those in the control $(3401 \pm 251$ vs $689 \pm 76,2329 \pm 216$ vs $753 \pm 97, \mathrm{p}<0.01)$. Postconditioning significantly reduced the release of CK and LDH from I/R myocardium $(2026 \pm 268$ vs $3401 \pm 251$, $1543 \pm 169$ vs $2329 \pm 216, p<0.01)$. These reduction were abolished by nor-BNI $(p<0.01)$. Regional myocardial I/R resulted in a significant increase in cardiomyocyte apoptosis $(18.7 \pm 2.5$ vs $1 \pm 0.25, \mathrm{p}<0.01)$. Postconditioning exerted a significant anti-apoptotic effect $(10.4 \pm 1.3$ vs $18.7 \pm 2.5, \mathrm{p}<0.01)$. This protective effect was attenuated by pretreatment with Nor-BNI ( $p>0.05)$. Immunoreactive dynorphin content $(\mathrm{pg} / \mathrm{ml})$ in serum significantly increased after postconditioning (78.5 \pm 12 vs $37.3 \pm 6.5, p<0.01)$. Increased dynorphin did not reduced by $\kappa$ opioid receptor antagonist Nor-BNI ( $p>0.05)$.

Conclusions We find that cardiac protection and anti-apoptotic effect of postconditioning is mediated by activating $\kappa$ opioid receptor. And cardiac protective and anti-apoptosis effect of postconditioning is mediated by enhanced dynorphin express in rats. Recently, clinical use of postconditioning as a treatment for cardiovascular disease has been an increasing attention, and opioid receptor triggers postconditoning, so the study of the relationship between $\kappa$ opioid receptor and ischaemia reperfusion injury (IRI) may provide a new insight for the curing of IRI.

\section{e0153 EFFECT OF PLATELET MICROPARTILES ON THE EXPRESSION OF CELL ADHESION MOLECULE IN ENDOTHELIAL CELL}

doi:10.1136/hrt.2010.208967.153

Yu Hui-ning, Cong Hong-liang, Li Ximing, Mao Yongmin. Tianjin Chest Hospital, Tianjin, China

Objective To examine the expressions of cell adhesion molecule (Eselectin.VCAM-1.ICAM-1) in HUVECs (CRL-1730), which is affected by platelet microparticles (PMPs). To investigate the effects of platelet microparticles in coronary heart disease.

Methods 1. PMPs was extracted from anticoagulated blood with sodium citrate. The purity of PMPs was measured by flow cytometry. 2. The prepared PMPs and CRL -1730 cell were co-cultured. The first part was divided into five groups based on the concentration of PMPs. The concentration were: $0,10 \mu \mathrm{g} / \mathrm{ml}, 30 \mu \mathrm{g} / \mathrm{ml}, 50 \mu \mathrm{g} / \mathrm{ml}$, $100 \mu \mathrm{g} / \mathrm{ml}$, each group contained four wells. Cells in wells were collected after $4 \mathrm{~h}$. The second part was didied into three groups based on the time of co-cultivation: $2 \mathrm{~h}, 4 \mathrm{~h}, 24 \mathrm{~h}$, and the concentration of PMPs that were added into each wells was $50 \mu \mathrm{g} / \mathrm{ml}$. Each group contained four wells. Cells in wells were collected after $2 \mathrm{~h}$, $4 \mathrm{~h}, 24 \mathrm{~h}$ respectively. 3 . The RNA of cells was extracted. Semiquantitate reverse transcription-PCR (SORT-PCR) was used to detect the relative expression of E-selectin, I CAM-1 and VCAM-1 respectively.

Results In this study, we found that cultured HUVEC (CRL-1730) expressed E-selectin, ICAM-1 and VCAM-1 mRNA in basic states. The expressed levels of E-selectin, ICAM-1 and VCAM-1 were increased when HUVEC (CRL-1730) were interfered by PMPs of centain concentration $(\mathrm{p}<0.05)$. But the PMPs stimulated HUVECs (CRL-1730) at different times, the expressions of E-selectin, ICAM-1 and VCAM-1 were of no diffeence ( $p>0.05)$.

Conclusions 1 . The high purity of PMPs were successfully prepared in the study. 2. The PMPs may increase the expressions of E-selectin, ICAM-1 and VCAM-1 on HUVEC (CRL-1730). It may explain a possible mechanism of PMPs in coronary heart disease.

\section{e0154 EXPRESSION OF TUMOUR NECROSIS FACTOR- $\alpha$ CONVERTING ENZYME AND TUMOUR NECROSIS FACTOR- $\alpha$ IN RATS WITH ALCOHOLIC CARDIOMYOPATHY}

doi:10.1136/hrt.2010.208967.154

Li Jianqiang, Li Weimin, Li Yue, Zhao Jiyi, Kong Yihui, Guo Hong, Xue Jingyi, Sheng Li, Gong Yongtai, Xue Hongjie. The First Affiliated Hospital of Harbin Medical University, Harbin, China

Purpose Chronic excessive consumption of alcohol causes ventricular remodelling and eventually leads to alcoholic cardiomyopahty (ACM). Tumour necrosis factor- $\alpha$ converting enzyme (TACE) has been identified to cleave membrane-bound tumour necrosis factor- $\alpha$ (TNF- $\alpha$ ) to soluble TNF- $\alpha$, which has crucial roles in ventricular remodelling. This study aimed to investigate the expression of TACE and TNF- $\alpha$, and their impacts on ventricular remodelling in rats with ACM

Methods 50 healthy male Wistar rats were randomly divided into a control group $(n=20)$ and an ACM group $(n=30)$. Animals in the ACM group were given $10 \%$ alcohol ad libitum as the drinking 